\title{
Hierarchical Support Vector Machine Based Heartbeat Classification Using Higher Order Statistics and Hermite Basis Function
}

\author{
KS Park, BH Cho, DH Lee, SH Song, JS Lee, \\ YJ Chee, IY Kim, SI Kim \\ Department of Biomedical Engineering, Hanyang University, Seoul, Republic of Korea
}

\begin{abstract}
The heartbeat class detection of the electrocardiogram is important in cardiac disease diagnosis. For detecting morphological QRS complex, conventional detection algorithm have been designed to detect $P, Q R S, T$ wave. However, the detection of the $P$ and $T$ wave is difficult because their amplitudes are relatively low, and occasionally they are included in noise. We applied two morphological feature extraction methods: higher-order statistics and Hermite basis functions. Moreover, we assumed that the QRS complexes of class $N$ and $S$ may have a morphological similarity, and those of class $V$ and F may also have their own similarity. Therefore, we employed a hierarchical classification method using support vector machines, considering those similarities in the architecture. The results showed that our hierarchical classification method gives better performance than the conventional multiclass classification method. In addition, the Hermite basis functions gave more accurate results compared to the higher order statistics.
\end{abstract}

\section{Introduction}

The electrocardiogram (ECG) is the signal of electrical activity of the heart and is the most important data to investigate heart diseases and conditions [1]. The QRS complex is the most important wave in the ECG waveform to interpret and decide whether the heartbeat belongs to the normal state or the arrhythmia state. For QRS complex recognition, the shape and morphological properties of the $\mathrm{P}, \mathrm{QRS}$, and $\mathrm{T}$ wave have been widely used to extract features. However, the $\mathrm{P}$ and $\mathrm{T}$ wave are often difficult to detect because their amplitudes are relatively low, and they are occasionally included in noise. Therefore, the feature extraction methods may have missing value problem, and thus may cause bad results in the automatic diagnosis [2, 3].

Moreover, when ECG data have unbalanced data distribution, the conventional multiclass classification method may give skewed results to the majority class. To solve this unbalanced distribution problem, many researchers have proposed resampling methods that duplicate or discard samples and then make equal distribution.. However, the classification rule or prediction model from these methods cannot represent the original population and guarantee the test performance [4].

In this study, we applied higher order statistics (HOS) and Hermite basis functions (HBF) to extract features from the QRS complex. We also adopted the hierarchical classification scheme to solve the unbalanced class distribution problem. Using the support vector machines $[11,12]$, we compared the feature extraction methods and classification methods to evaluate the generalization performance.

\section{Methods}

\subsection{Data acquisition and preprocessing}

We used the MIT-BIH arrhythmia database include 48 recordings, which contains the two-channel 30-min ECG signals. There are approximately 110,000 heartbeats, and each of the beats is annotated as 15 different heartbeat classes [5]. Following the Association for the Advancement of Medical Instrumentation (AAMI) recommendations, we selected 44 recordings (the four recordings containing paced beats were removed) and combined the MIT-BIH heartbeat types into five heartbeat classes in this study. N-class includes beats originating in the sinus node (normal and bundle branch block beat types), S-class supraventricular ectopic beats, and V-class ventricular ectopic beats (VEBs). F-class includes beats that result from fusing normal and VEBs, and Q-class includes unknown beats including paced beats [6]. The Q-class was excluded in this research because the Q-class has very few data. We removed the baseline wondering by two median filters as a preprocessing. 
Table 1. Organized Dataset 1(DS1) and Dataset 2(DS2) from MIT-BIH dataset

\begin{tabular}{cccccc}
\hline \hline & $\mathrm{N}$ & $\mathrm{S}$ & $\mathrm{V}$ & $\mathrm{F}$ & Total \\
\hline DS1 & 45,868 & 943 & 4,259 & 415 & 51013 \\
(Ratio, \%) & $(89.08)$ & $(1.83)$ & $(8.24)$ & $(0.81)$ & $(100)$ \\
DS2 & 44,259 & 1,837 & 3,221 & 388 & 49705 \\
$($ Ratio, \%) & $(89.03)$ & $(3.71)$ & $(6.48)$ & $(0.78)$ & $(100)$ \\
\hline \hline
\end{tabular}

We divided the 44 recordings into two datasets; 22 recordings were contained in each dataset with the same approximate ratio of heartbeat classes. Each dataset has almost 50,000 heartbeats with arrhythmia recordings [2].

\subsection{Higher order statistics (HOS)}

We calculated the $2 \mathrm{nd}$, 3rd, and 4th order cumulants of each heartbeat signal using higher order statistics (HOS) methods. The cumulants are coefficients of the Taylor expansion of the cumulant-generating function [3]. They can be represented in terms of the statistical moments by their linear or nonlinear combinations. When $x(t)$ is the zero mean statistical process, the $2 \mathrm{nd}$, 3rd, and 4 th order cumulants are equal to the corresponding moments [7]. The 2nd order cumulant expresses the autocorrelation of the signal, the 3 rd order cumulant expresses the skewness, and 4th order cumulant expresses to and kurtosis of a zero mean signal. We took $250 \mathrm{~ms}$ (90 sample points) before and after the R peak in an ECG beat. Using the 181 sample points, the $2 \mathrm{nd}$, 3rd, and 4th order cumulants were calculated. Figure 1 shows an example of the cumulants; (a) represents an ECG waveform, (b) the second cumulant, (c) the third cumulant, and (d) the fourth cumulant of the original waveform. If all the
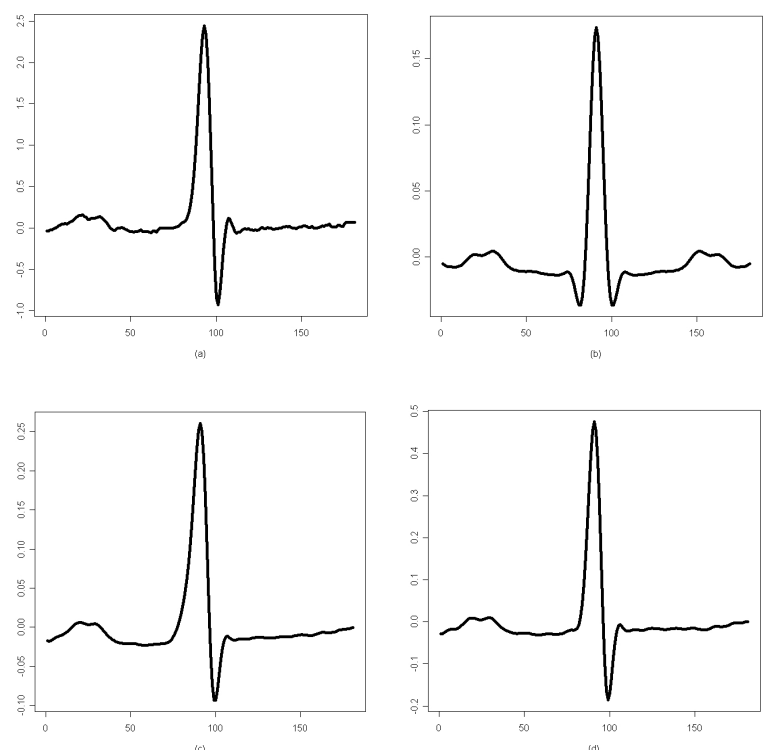

Figure 1 (a) The N-class waveform of the ECG beat and its cumulant representations of the (b) second, (c) third, and (d) fourth orders

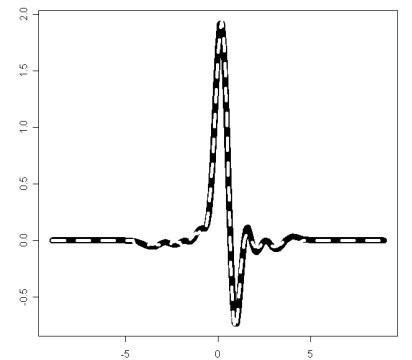

Figure 2 The normal QRS complex of an ECG beat (black solid line) and its estimation using a Hermite polynomial of the $20^{\text {th }}$ order (white dashed line)

results of the cumulants are used as the feature vector for classification, the testing process may have a poor performance due to the overfitting problem. To avoid this problem, we chose ten sample points of each cumulant as features. The sample points of 15, 30, 45, 60, 75, 90, 105, 120,135 , and 150 were chosen to represent the cumulants of the QRS complex.

Additionally, we extracted three interval features; the RR interval of the heartbeat, the mean RR interval of the ten last beats, and the ratio between the RR interval and the mean RR interval.

\subsection{Hermite basis functions (HBF)}

Hermite basis functions ( $\mathrm{HBF}$ ) are very similar to the Fourier series. The HBF can produce new signals with the functions' various waveform combinations and thus approximate the QRS complex of the ECG. The features distinguishing the shape of the ECG are formed from the coefficients of the HBF expansion. The higher the order of the function, the higher its frequency of changes within the time window and the better its capability to reconstruct the ECG pattern. By increasing the order of the model, the approximation error may become smaller.

Nevertheless, higher order models need more computation cost and may cause overfitting problem in generalization performance. Using singular value decomposition and the pseudoinverse technique, we solved the linear combination of Hermite basis function [8-10].

In this research, the order of the Hermite polynomial was set to 20 to optimize the features. We also took 250 ms (90 sample points) before and after the $\mathrm{R}$ peak in the ECG beat. In the figure 2, solid line is an original heartbeat signal, and dashed line is the approximation of the original signal by the 20th order Hermite polynomial.

\subsection{Hierarchical classification}

We assumed that the QRS complexes of classes $\mathrm{N}$ and $\mathrm{S}$ may have a morphological similarity and those of classes $\mathrm{V}$ and $\mathrm{F}$ may have difference compared to those 


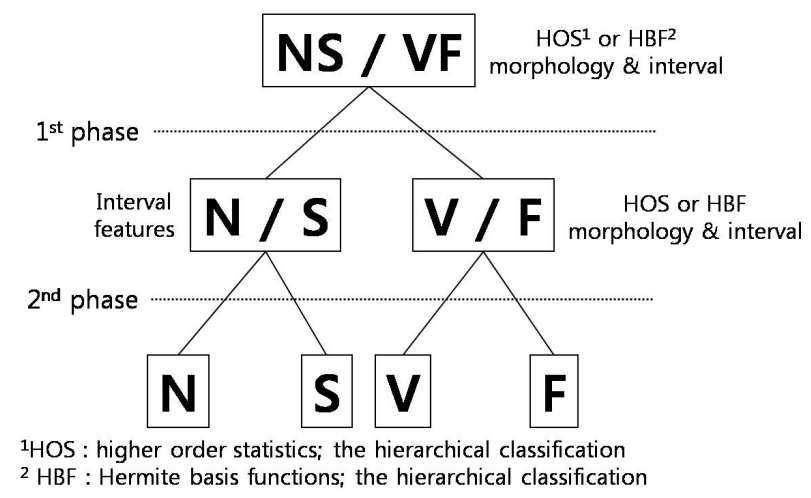

Figure 3 The schematic of hierarchical classification

of class $\mathrm{N}$ and $\mathrm{S}$. Therefore class $\mathrm{N}$ and $\mathrm{S}$ were bounded in one group, and class $\mathrm{V}$ and $\mathrm{F}$ were bounded in another group. To classify classes $\mathrm{N}$ and $\mathrm{S}$, we used the RR interval features, because the interval features were more related than the morphology. Figure 3 shows the schematic of our hierarchical classification.

\subsection{Experimental procedure}

We assumed that the QRS complexes of class $\mathrm{N}$ and $\mathrm{S}$ may have a morphological similarity, and those of class $\mathrm{V}$ and $\mathrm{F}$ may also have their own similarity. Therefore, we employed a hierarchical classification method using support vector machines, considering those similarities in the architecture. Figure 3 shows the schematic of our hierarchical classification. In the first phase, we initially classify the sample heartbeat into one of the group (NS class vs. VF class) using HOS, HBF, and interval features. To classify class $\mathrm{N}$ and $\mathrm{S}$ in the second phase, we used only the RR interval features because the interval features were more related than the morphology in this classification. For the class $\mathrm{V}$ and $\mathrm{F}$ in the other second phase, we also used the whole features.

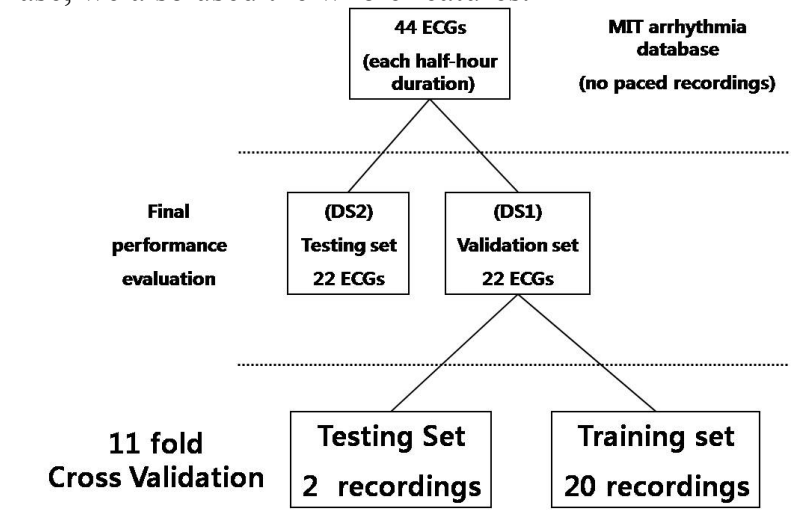

(Not totally random $\mathrm{CV}$ )

Figure 4 Division of the validation set (DS1) into training and test set using 11 fold cross validation. A Final performance evaluation is performed with testing set (DS2)
Table 2 Classification performance of the conventional multiclass classification and the hierarchical classification on validation set (DS1)

\begin{tabular}{ccccc}
\hline \hline Parameter & mHOS & $\mathrm{mHBF}^{2}$ & $\mathrm{hHOS}^{3}$ & $\mathrm{hHBF}^{4}$ \\
\hline N sensitivity & 89.82 & 90.67 & 87.97 & 88.96 \\
S sensitivity & 0.11 & 0.11 & 47.30 & 89.29 \\
V sensitivity & 68.00 & 68.97 & 85.70 & 92.25 \\
F sensitivity & 0.24 & 0.00 & 59.76 & 64.82 \\
Accuracy & 85.81 & 86.65 & 86.81 & 89.04 \\
Mean value of & 38.54 & 39.94 & 70.18 & 83.83 \\
sensitivities & & & \\
\hline \hline
\end{tabular}

${ }^{1}$ mHOS : higher order statistics; the conventional multiclass classification

${ }^{2}$ mHBF : Hermite basis functions; the conventional multiclass classification

${ }^{3}$ hHOS : higher order statistics; the hierarchical classification

${ }^{3} \mathrm{hHBF}$ : Hermite basis functions; the hierarchical classification

\section{Results}

Using DS1 and 11 fold cross validation, the results are shown in Table 2 for the different feature extraction and classification methods. In the aspect of accuracy, there was no remarkable difference between the conventional multiclass classification and our hierarchical method. For the conventional multiclass classification method, the classification accuracies were $85.81 \%$ and $86.65 \%$ for feature extraction methods of HOS and HBF, respectively. For the hierarchical classification method, accuracies were $86.81 \%$ and $89.04 \%$ for HOS and HBF, respectively.

On the other hand, for the mean value of sensitivities, those values for the hierarchical classification methods $(70.18 \%$ and $83.83 \%)$ were higher than those for the conventional multiclass classification (38.54 \%, 39.94\%).

Table 3 presents the results of the final test performance using DS2. In the aspect of accuracy, the conventional multiclass classification seems to be superior to our hierarchical classification method. For the

Table 3 Classification performance of the hierarchical classification and the conventional multiclass classification on test set (DS2)

\begin{tabular}{ccccc}
\hline \hline Parameter & mHOS & $\mathrm{mHBF}^{2}$ & $\mathrm{hHOS}^{3}$ & $\mathrm{hHBF}^{4}$ \\
\hline N sensitivity & 99.65 & 99.38 & 81.23 & 86.25 \\
S sensitivity & 0.00 & 0.00 & 57.65 & 82.63 \\
V sensitivity & 84.48 & 65.48 & 83.11 & 80.88 \\
F sensitivity & 0.52 & 15.46 & 79.90 & 54.90 \\
Accuracy & 94.21 & 92.86 & 80.47 & 85.56 \\
Mean value of & 46.16 & 45.08 & 75.47 & 76.16 \\
sensitivities & & &
\end{tabular}

${ }^{1}$ mHOS : higher order statistics; the conventional multiclass classification

${ }^{2} \mathrm{mHBF}$ : Hermite basis functions; the conventional multiclass classification

${ }^{3}$ hHOS : higher order statistics; the hierarchical classification

${ }^{3} \mathrm{hHBF}$ : Hermite basis functions; the hierarchical classification 
conventional multiclass classification method, the classification accuracies were $94.21 \%$ and $92.86 \%$ for feature extraction methods of HOS and HBF, respectively. For the hierarchical classification method, on the other hand, were $80.47 \%$ and $85.56 \%$ for HOS and $\mathrm{HBF}$, respectively.

However, the mean values of sensitivity for the hierarchical classification methods $(75.47 \%$ and $76.16 \%$ ) were higher than those for the conventional multiclass classification $(46.16 \%, 45.08 \%)$.

\section{Discussion and conclusions}

The goal of this research is to classify the multiclass ECG heartbeats with the unbalanced data distribution. In order to tackle the problem, we employed a hierarchical classification method that takes advantage of domain knowledge in the hierarchy architecture: the morphological similarities of the heartbeat classes. The conventional multiclass classification method could not classify specific classes well, such as supra-ventricular ectopic beat and fusion beat. On the other hand, our hierarchical classification method showed better classification performance than the conventional multiclass classification method. Despite the loss in accuracy and sensitivities of class $\mathrm{N}$ and $\mathrm{V}$, hierarchical classification improved the mean values of sensitivity. This means our classification method can distinguish the multiclass heartbeats with the unbalanced data distribution well.

In the hierarchical classification, the HBF gave more accurate results compared to the higher order statistics. Moreover, the HBF had a more balanced sensitivity performance results, meaning the $\mathrm{HBF}$ is more feasible in the ECG feature extraction and classification.

We used all feature vectors for classification in this research. In the future work, we have a plan to employ other feature extraction methods and the feature selection method to exclude unnecessary extra features.

\section{Acknowledgements}

This study was supported by a Silver Technology Development Project, Ministry of Knowledge Economy, Republic of Korea.

\section{References}

[1] $\mathrm{Hu} \mathrm{YH}$, Palreddy S, Tompkins W. A patient adaptable ECG beat classifier using a mixture of experts approach. IEEE Trans Biomed Eng 2007;44:891-900

[2] Chazal P, O'Dwyer M, Reilly R. Automatic Classification of Heartbeats Using ECG Morphology and Heartbeat Interval Features. IEEE Trans Biomed Eng 2004;51:11961206

[3] Osowski S, Hoai LT, arkiewicz T. Support vector machine-based expert system for reliable heartbeat recognition. IEEE Trans Biomed Eng 2004;51:582-89

[4] Elkan C. The foundation of cost-sensitive learning. 17th International Joint Conference on Artificial Intelligence 2001:973-78

[5] Mark R, Mody G. MIT-BIH Arrhythmia Database. 1997 Online available: http://www.physionet.org/

[6] American National Standard. Testing and reporting performance results of cardiac. ANSI/AAMI EC57 : 1998/(R)2003

[7] Nikias C, Petropulu A. Higher Order Spectral Analysis. Prentice-Hall, 1993

[8] Lagerholm M, Peterson C, Braccini G, Edenbrandt L, and Sornmo L. Clustering ECG complexes using Hermite functions and self-organizing maps. IEEE Trans Biomed Eng 2000;47:838-47

[9] Sansone G. Orthogonal Functions. Dover, 1991

[10] Golub G, Van Loan C. Matrix Computations. Johns Hopkins University, 1991

[11] Vapnik V. Statistical Learning Theory. Wiley, 1998

[12] Cho B, Lee J, Chee Y, Kim K, Kim I, Kim S. Prediction of Diabetic Nephropathy from Diabetes Database Using Feature Selection Methods and SVM Learning. Kor Society of Medical \& Biological Eng 2007;28:255-62

Address for correspondence

Beak Hwan Cho

Dept. of Biomedical Eng., Hanyang university, Seoul 133-791, Republic of Korea

uranus@bme.hanyang.ac.kr 